\title{
PCB Board Design of Switching Power Supply with High Power Factor in Communication Equipment
}

\author{
XI FENG ${ }^{1, a^{*}}$, YUXUAN SUN ${ }^{2, b^{*}}$ \\ ${ }^{1}$ Networking Academy, Haikou College of Economics, Haikou City, Hainan, China \\ ${ }^{2}$ Networking Academy, Haikou College of Economics, Haikou City, Hainan, China \\ afengxi_000@qq.com, bsunyuxuan09@163.com
}

Keywords: PCB board high power factor switching power supply

Abstract. In the design of switching power supplies of high power factor in communication devices, high-quality PCB board design is the most significant aspect in solution to electromagnetic compatibility and reliability of the entire system[1]. The article introduces the principles and methods of PCB board design in system design and optimization, as well as scientific rational distribution and how to deal with the key technical measures. This system meets the design requirements after commissioned.

\section{Introduction of PCB Design}

PCB board, printed circuit board invented by the Austrian Paul Eisler, aims to achieve circuit designers' functional requirement based on circuit schematics. PCB design mainly refers to the layout design, which needs to consider layouts of various factors such as external connections, internal electronic components, metal wires, vias and heat dissipation. Simple PCB design can be done manually, while complex needs computer-aided design and implementation such as CAD software etc.. The softwares commonly used to design PCB are protel, protel 99se, protel DXP, Altium etc.. PCB board can be divided into three categories according to the number of layers: single-sided, double-sided and multilayer three. The switching power supply is generally designed as a single panel taking into account the low circuit complexity and cost.

\section{Principles of PCB Design}

Excellent PCB board design not only reduces production costs, but also achieves good circuit performance and heat dissipation. The characteristic impedances of controlled impedance boards and circuit in design are the most important and common problems. PCB board design is the continuation and improvement of a circuit schematic design, whose unreasonable design will cause its performance greatly reduced, or even not work. So good PCB board design is an important part of the circuit function implemented.

PCB board design generally abide by the following aspects:

1. Overall Layout

1) Consider noise, coupling, impedance and other factors, according to different functions and signals, divide circuit board area into different modules and components reasonably and arrange components according to their electrical characteristics;

2) If necessary, plan the size and specifications of the printed circuit board as well as the number of layers; 
3) Consider the needs of production, testing, maintenance and post-improvement;

4) Based on necessary, make sure whether the printed circuit board need to be coppered, or grounded.

2. Component Layout

1) Elements' layout should facilitate the signal flow, keeping the same direction with the signal;

2) Ensure the electrical characteristics as the premise, the components should be placed orderly and neat with the shortest connection between the various components wire;

3) Place core elements and structure components of fixed position first generally, then large and special ones, and others at last;

4) Components on printed circuit boards should be evenly distributed, density consistent with no core bias;

5) Pay attention components' heat dissipation, high heat-sensitive components not placed next to the ones hot easily;

6) Under high frequency situation, also need to attention to the impact between the elements and eliminate electromagnetic interference.

3. Traces Layout

1) Apply automatic and manual routing wire methods combining, with corner traces as greater than 90 degrees as possible;

2) Reasonable the width of the wiring, uniform arrangement, less use vias or fly line jumper;

3) As thick as possible power and ground lines, the shorter and thicker the signal line the better, the thinner and shorter the high-frequency digital circuit lines; large current and high voltage signals as thick as possible;

4) Attention space between lines, for example: interference between the large current signals, high voltage signals and small signals with each other, affecting the quality of the signal;

5) When double-sided alignment, try to make sure both sides of wires vertical, avoid parallel to each other, resulting in parasitic coupling.

\section{Switching Power Supply PCB Design}

A good switching power supply PCB design is as important as its circuit design. Good PCB design ensures various technical indicators in a switching power supply, such as: electrical performance, safety performance indicators, electromagnetic compatibility indicators to make it stable, easy to install components, and ongoing maintenance.

\section{Ground}

In considering the design of switching power supply circuit board, an experienced designer will carefully consider the circuit grounding method to obtain stable power supply. But in actual design it is difficult to get a perfect grounding scheme, because any grounding design will directly affect the circuit performance. If a longer lead directly to the various components on the circuit is connected to the negative terminal of the power supply or battery, this line represents the ground floor or the ground, which has resistance and inductance. When the current goes through, reflux power produces, resulting in corresponding pressure drop, and therefore the ground loop is not ideal power value.

Ground line, as a reference potential point circuit or system, is common conductor of each circuit, current in any circuit or system goes through which to form as ground current. However, any conductor has certain impedance, while a current passes, a voltage would surely be generated. Hence it is necessary to design a good ground system to reduce the potential difference as well as to solve EMI problems. 
Working electrical level of small current signal is low, particularly vulnerable to electromagnetic interference, which causes circuit failure or circuit performance degradation phenomenon. While operating current of large current signal is large, relatively speaking, the current of its grounding conductor is large, easily causing interference to the small-signal circuit because of the coupling action of the ground wire, affecting its normal work. At the moment of switching transistor instant turning on and off in switching power supply, dramatic changes happen in the voltage and current, prone to serious interference on the ground line, coupled with directly complex relationships: the high frequency, measuring, controlling and driving circuit, thus designing the ground layout cannot just complete "dc potential gang" concept [2]. Whether ground layout is reasonable can be measured according to the following two conditions.

1) Whether current in ground line flows through other unrelated circuit, conductors or components;

2) Whether there is current of other circuit flowing into the ground in this part of the circuit.

2. The overall layout

In PCB layout of switching power supply the location of the functional modules should be arranged in accordance with the circuit diagram. According to the signal flow direction: AC input filter, high-voltage rectifier filter, high-frequency inverter part, low-voltage output, such layouts making it consistent with the signal direction, the smooth flow of the signal, also to facilitate post-checking and debugging.

AC input and DC output parts of the switching power supply should be isolated, not too close to prevent electromagnetic interference. Components of high-pressure region and low should be placed separately to avoid creeping power. Traces of control circuits and power circuits should be separated, single-point grounding to connect each other's ground loops together be best. The control circuit part in switching power supply is compatibly poor reliability and stability part, more sensitive to electromagnetic interference. Therefore, a large area grounding method cannot be used because it is easy to introduce electromagnetic interference as far as possible to narrow the area surrounded by the circuit to reduce the "receiving antenna" the size of the reception interference, improving the reliability of the switching power supply and electromagnetic compatibility [3].

3. Component Layout

In PCB design, electronic components are generally installed in horizontal and vertical two installation methods. For the same piece of $\mathrm{PCB}$, mounting components should be consistent. Bulky components should be in horizontal installation, avoiding excessive height and vibration performance deterioration. For heavy quality components, bracket needs to be adopted. For easy heating, heavy quality, large volume components, the heat sink should be considered to be added to, and arranged on the outside of the PCB.

After planning functional areas ready, first the core components are placed, while other components surrounding core components of the layout, shorten the wiring distance between the various components. For some high voltage, large current, high-frequency electronic components or circuit, be careful with their distance from others to avoid accidental short circuits and interference caused by high-voltage discharge.

\section{This System's PCB Design}

The system is first divided into different modules according to different functions when designing PCB board. At last in the debugger, first use of Dupont lines, the connection relationship will be determined finally after successful commissioning. In order to implement overall system function, the main circuit of the printed circuit board design is the significant part to notice, which 
includes a PFC control circuit, the rectifier filter circuit and Boost circuit. The following aspects are important factors in PCB Design. ( shown in Fig. 1, Fig. 2)

First, UCC28019 chip functions as the core PFC control circuit, while the layout and wiring around the element are particularly important;

Second, PFC control circuit is small current signals, while Boost circuit and the rectifier filter circuit is large, as a result of which processing ground connection between these two signals is also very important;

Third, at high frequency, it is prone to generate resistance and inductance, thus affecting the frequency response;

Fourth, the large current signal is prone to generate heat, and should be assured the distance between the components;

Fifth, the layout should facilitate the signal flow in the same direction as much as possible to keep the signal flow.

When drawing the PCB, it should make peripheral components as close to the UCC28019 chip, and the ground lines of capacitances connected to its pins should be connected together directly beneath the chip; connect ground lines of boost and rectifier filter circuit together, as thick as possible, try to connect ground of signal with ground of main circuit together in the E pole of switching transistor, avoiding large current signal and low current signal collision with the impact of the chip work, easy for signal circulation, forming a loop. Traces of large current signals must be chosen as thick as possible, also increasing the heat sink for some elements easy to heat, and pay attention to the flow of their signals to form a loop.

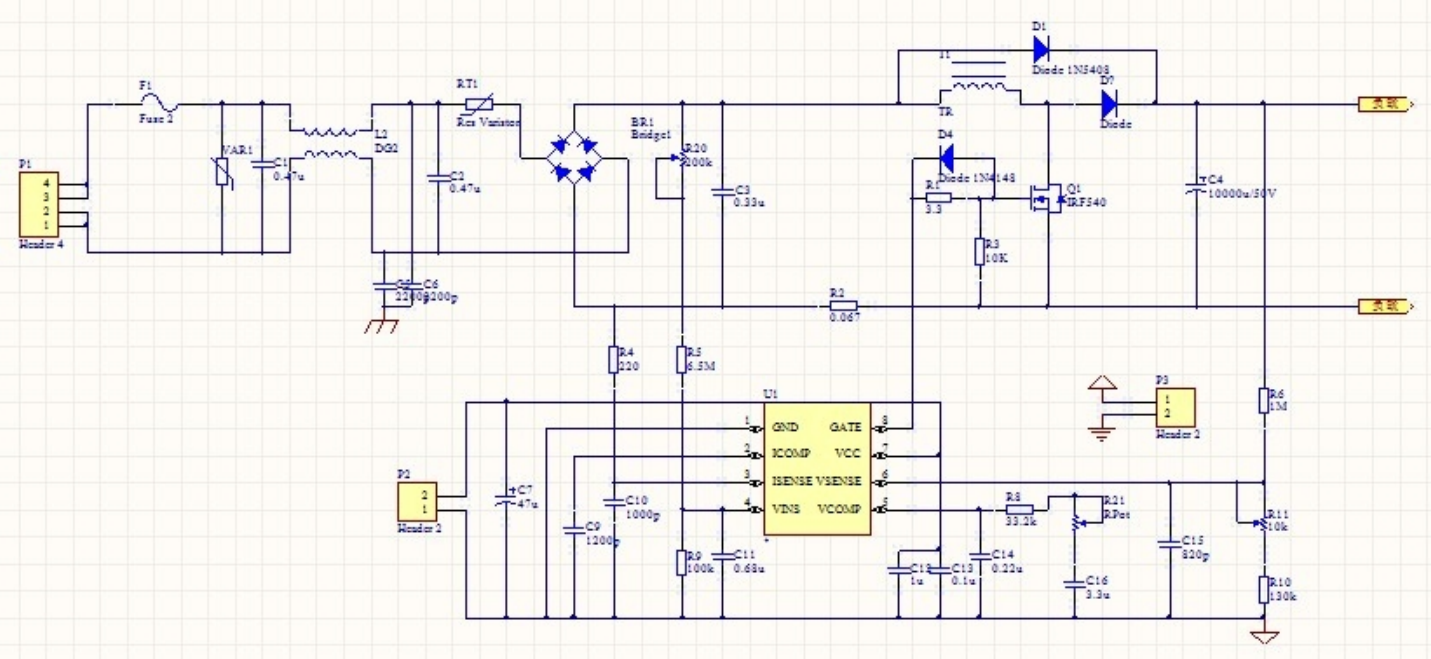

Fig. 1 High power factor switching power supply main circuit diagram 


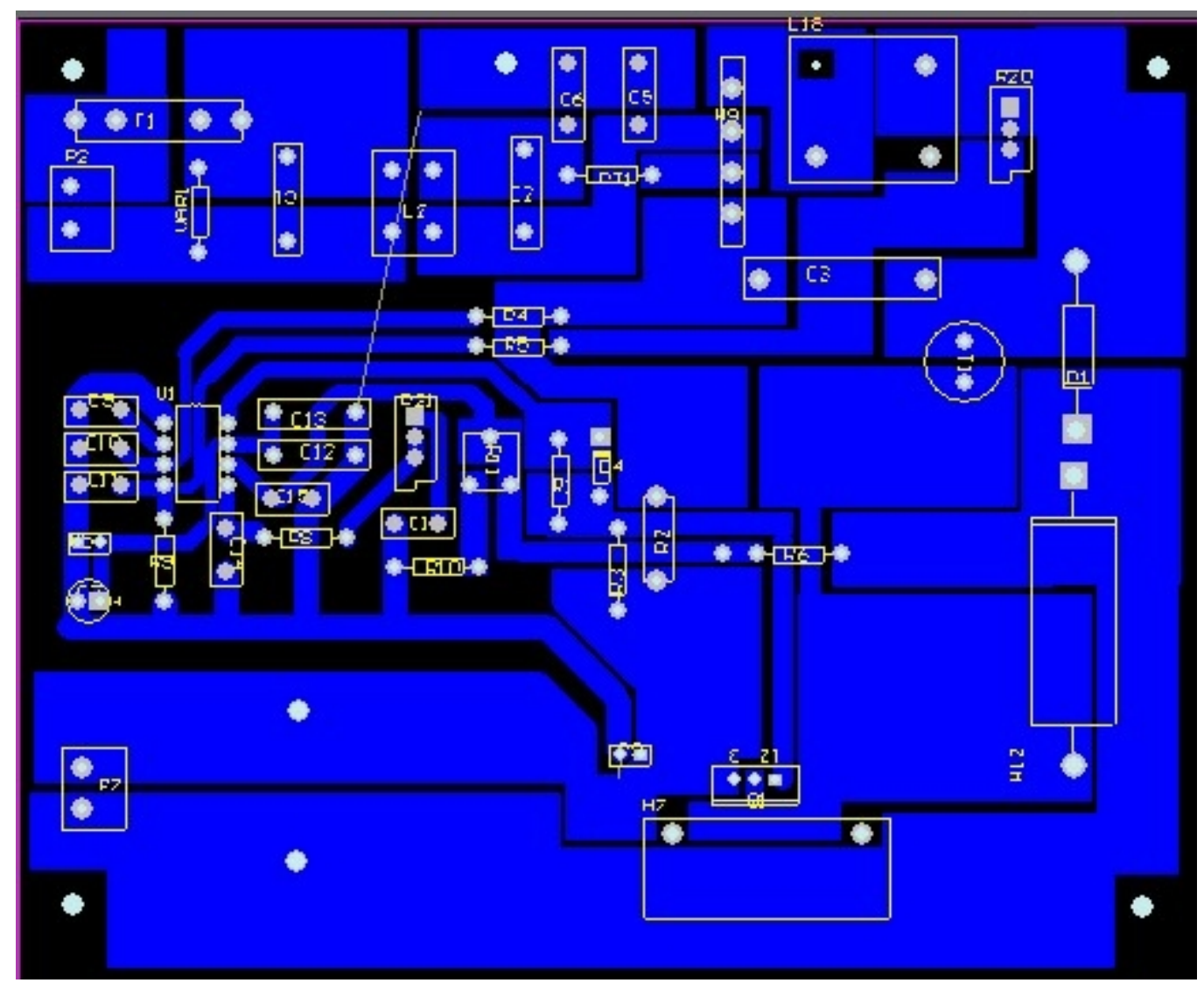

Fig. 2 High power factor switching power supply PCB board diagram

\section{Conclusions}

The communication power supply module in the entire communications industry is of a relatively small percentage, but it is the key decision factor to judge a communication system's quality whether good or bad, a complete also irreplaceable separate product in communications network. For low power factor and harmonic pollution problems in conventional switching power supply, the system is designed as a high power factor switching power supply based on UCC28019 chip. As a result of several new technologies and enhancing the design technologies (mainly the optimization of the PCB), the design is with the advantage of a high power factor, high efficiency, low harmonics and low noise. After complement the system has achieved $2^{\text {nd }}$ National Prize in the 2013 National Undergraduate Electronic Design Contest as an undergraduate group.

\section{Acknowledgements}

This article Haikou College of Economics teach key research projects (No. hjkz14-08) funded content.

\section{References}

[1] Song Fu, Root frequency switching power supply Active EMI Filter applied research, J. Electrical Equipment. 04 (2009) 31-33.

[2] Wei Xuguang, Chen Jianfeng, High power factor switching power supply communication power technology t. 26 (2009) 28-30.

[3] Huang Zhiwei, Printed circuit board (PCB) design techniques and practice, second ed., Beijing, 2012. 